\title{
Chemical biology of natural indolocarbazole products: 30 years since the discovery of staurosporine
}

\author{
Hirofumi Nakano and Satoshi Ōmura
}

Staurosporine was discovered at the Kitasato Institute in 1977 while screening for microbial alkaloids using chemical detection methods. It was during the same era that protein kinase $C$ was discovered and oncogene v-src was shown to have protein kinase activity. Staurosporine was first isolated from a culture of Actinomyces that originated in a soil sample collected in Mizusawa City, Japan. Thereafter, indolocarbazole compounds have been isolated from a variety of organisms. The biosynthesis of staurosporine and related indolocarbazoles was finally elucidated during the past decade through genetic and biochemical studies. Subsequently, several novel indolocarbazoles have been produced using combinatorial biosynthesis. In 1986,9 years since its discovery, staurosporine and related indolocarbazoles were shown to be nanomolar inhibitors of protein kinases. They can thus be viewed as forerunners of today's crop of novel anticancer drugs. The finding led many pharmaceutical companies to search for selective protein kinase inhibitors by screening natural products and through chemical synthesis. In the 1990s, imatinib, a Bcr-Abl tyrosine kinase inhibitor, was synthesized and, following human clinical trials for chronic myelogenous leukemia, it was approved for use in the USA in 2001. In 1992, mammalian topoisomerases were shown to be targets for indolocarbazoles. This opened up new possibilities in that indolocarbazole compounds could selectively interact with ATPbinding sites of not only protein kinases but also other proteins that had slight differences in ATP-binding sites. ABCG2, an ATP-binding cassette transporter, was recently identified as an important new target for indolocarbazoles.

The Journal of Antibiotics (2009) 62, 17-26; doi:10.1038/ja.2008.4; published online 9 January 2009

Keywords: biosynthesis; indolocarbazole; protein kinase; staurosporine; topoisomerase

\section{INTRODUCTION}

The discovery of medically useful natural products has heralded hitherto unimagined possibilities in the chemotherapy of human and animal diseases. . $2,3^{2}$

It is well known that important medical compounds, such as penicillin, cyclosporine A and lovastatin, were only developed as drugs once their key properties were recognized, more than 10 years after their initial discovery. ${ }^{4}$ Similarly, in the case of staurosporines, their crucial protein kinase inhibitory properties were only identified a decade or so after their initial discovery. ${ }^{5}$

In 1986, 9 years after the isolation of staurosporine from a streptomyces, the related natural indolocarbazole products, staurosporine and $\mathrm{K} 252$, were shown to be nanomolar inhibitors of protein kinases, offering tremendous promise for drug development. ${ }^{6}$ The reports led many pharmaceutical companies to begin searching for selective protein kinase inhibitors through natural product screening and chemical synthesis, with the result that, during the 1990s, protein kinases became the second most important drug target after G-protein-coupled receptors. ${ }^{7}$

In parallel with the development of indolocarbazoles as anticancer drugs targeting protein kinases, mammalian DNA topoisomerase I was shown to be a new target for indolocarbazoles by Yamashita et al. ${ }^{8}$ Thereafter, many antitumor indolocarbazoles have been synthesized, as DNA topoisomerases were known to be targets for antitumor drugs such as camptothecin and VP-16. DNA topoisomerases alter DNA topology by transiently breaking and re-sealing one strand of DNA through a covalent protein-DNA intermediate. $^{9}$ In 1996, it was shown that topoisomerase I has an intrinsic protein kinase activity (Topo I kinase) required for phosphorylation of the SR (serine arginine-rich) protein required for splicing. ${ }^{10}$

The action of indolocarbazole derivatives on topoisomerase indicated that these compounds may selectively interact with ATPbinding sites of not only protein kinases but also other proteins. As an example of this, during the current decade, it was shown that ABCG2, an $\mathrm{ABC}$ transporter with importance in drug resistance, oral drug absorption and stem cell biology, could be a key new target for indolocarbazoles.

This review outlines the pivotal pioneering studies relating to the discovery, biosynthesis and biological activities of natural indolocarbazole products. 


\section{PRODUCING ORGANISM}

Staurosporine was discovered in 1977 in a culture of an actinomycete (Streptomyces strain AM-2282 ${ }^{\mathrm{T}}$ ) while screening for microbial alkaloids using chemical detection methods ${ }^{11}$. The strain AM- $2282^{\mathrm{T}}$ (NRRL 11184, ATCC 55006) has been renamed through repeated revisions of the taxonomy of soil Actinomyces as Streptomyces staurosporeus AM-2282 $2^{\mathrm{T}}$ in 1977, Saccharothrix aerocolonigenes subsp. staurosporea AM-2282 $2^{\mathrm{T}}$ in $1995^{12}$ and Lentzea albida in $2002 .{ }^{13}$ Over the past 30 years, staurosporine and related natural indolocarbazole compounds have been isolated from several actinomycetes (including Streptomyces, Saccharothrix, Lentzea, Lechevalieria, Nocardia, Nocardiopsis, Nonomuraea, Actinomadura and Micromonospora) as well as from myxomycetes (slime molds) and cyanobacteria (Figure 1).

Staurosporine derivatives have also been isolated from marine invertebrates, such as sponges, tunicates, bryozoans and mollusks. However, it remains unknown whether invertebrates actually have
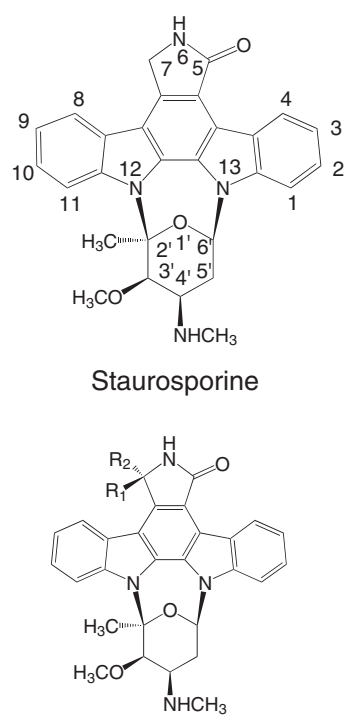

UCN -01 $\left(\mathrm{R}_{1}=\mathrm{OH}, \mathrm{R}_{2}=\mathrm{H}\right)$

UCN -02 $\left(\mathrm{R}_{1}=\mathrm{H}, \mathrm{R}_{2}=\mathrm{OH}\right)$

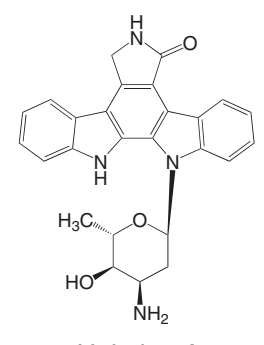

Holyrine A

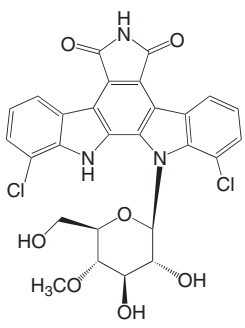

Rebeccamycin

Figure 1 Chemical structures of staurosporine and related indolocarbazoles isolated from culture broths. genes for indolocarbazole biosynthesis, as many natural products from marine invertebrates are produced by associated microorganisms. ${ }^{14}$

Interestingly, half of the 14 indolocarbazole-producing strains deposited in the global culture collection have been isolated from Japanese soils. In the 1980 s, fermentation broths of 5163 new Japanese soil isolates were tested and five Streptomyces were found to produce staurosporine, together with new analogs (UCN-01 and UCN-02 (stereo-isomers of 7-hydroxy staurosporines)). In other words, ca $0.1 \%$ of newly isolated soil actinomycetes were shown to produce staurosporine using a fixed culture condition. ${ }^{15}$

In 1993, staurosporine and K252a were shown to inhibit in vitro phosphorylation of crude extracts from Streptomyces griseus and also from a staurosporine-producing Streptomyces sp. ${ }^{16}$ Although staurosporine did not show significant antibacterial activity, it was shown to affect cell differentiation processes in Streptomycetes, such as pigment production and spore formation, depending on the AfsK family serine/threonine protein kinases involved. Later, on the basis of genome sequence analysis of Streptomyces avermitilis in $2001^{17}$ and Streptomyces coelicolor in 2002, ${ }^{18}$ it was revealed that more than 30 protein kinase genes are coded in these organisms. Further research is needed to determine the exact role and impact of staurosporine on differentiation of producing strains and microorganisms in soil.

\section{BIOSYNTHESIS OF STAUROSPORINE}

Biosynthetic studies carried out in the 1980s and 1990s using isotopelabeled precursors showed that the indolocarbazole structure of staurosporine is derived from two molecules of tryptophan, and that the sugar moiety is derived from glucose and methionine. Cloning of the biosynthetic genes of staurosporine was triggered in 2000 by identification of the $n g t$ gene encoding $N$-glycosyltransferase. Ohuchi et al. ${ }^{19}$ heterologously expressed the $n g t$ transferase gene from Lechevalieria aerocolonigenes, a rebeccamycin producer, in Streptomyces lividans and showed that $n g t$ is responsible for $\mathrm{N}$-glycosylation of the indolocarbazole chromophore. Starting from the ngt gene, whole biosynthetic gene clusters of staurosporine and rebeccamycin have been cloned by Onaka et al. ${ }^{20,21}$ and Sanchez et al..$^{22}$ To date, structures of the accumulated products from 18 single-gene disruption mutants of staurosporine and rebeccamycin biosynthesis gene clusters have been identified (Figure 2).

Studies of these accumulated products and the gene function predicted by the amino-acid sequence database searches have revealed the biosynthetic pathway of staurosporine and rebeccamycin. ${ }^{14,23}$ (Figure 3).

In staurosporine biosynthesis, staO initiates synthesis by catalyzing L-tryptophan to the imine form of indole-3-pyruvic acid (IPA imine) and staD, and then catalyzes the coupling of two IPA imines to yield chromopyrrolic acid. Formation of the indolocarbazole core of staurosporine is catalyzed by staP, which converts chromopyrrolic acid into three indolocarbazole compounds, staurosporine aglycone (K252c), 7-hydroxy-K252c and acryriaflavin A, by intramolecular C-C bond formation and oxidative decarboxylation. Crystallography of $\mathrm{P} 450$ staP revealed that a heme of staP removes two electrons from the indole ring to generate an indole cation radical, and intramolecular radical coupling then forms the $\mathrm{C}-\mathrm{C}$ bond to yield the indolocarbazole core. ${ }^{24}$ The presence of staC predominantly directs the formation of K252c. staG catalyzes N-glycosidic bond formation between N-13 and $\mathrm{C}-6^{\prime}$ and then staN, a P450 homolog, catalyzes an additional $\mathrm{C}-\mathrm{N}$ bond formation between $\mathrm{N}-12$ and $\mathrm{C}-2^{\prime}$. These two enzymes convert $\mathrm{K} 252 \mathrm{c}$ to $3^{\prime}$-O-demethyl, $4^{\prime}$ - $\mathrm{N}$-demethyl-staurosporine through holyrine A and holyrine B. staMA catalyzes $N$-methylation 

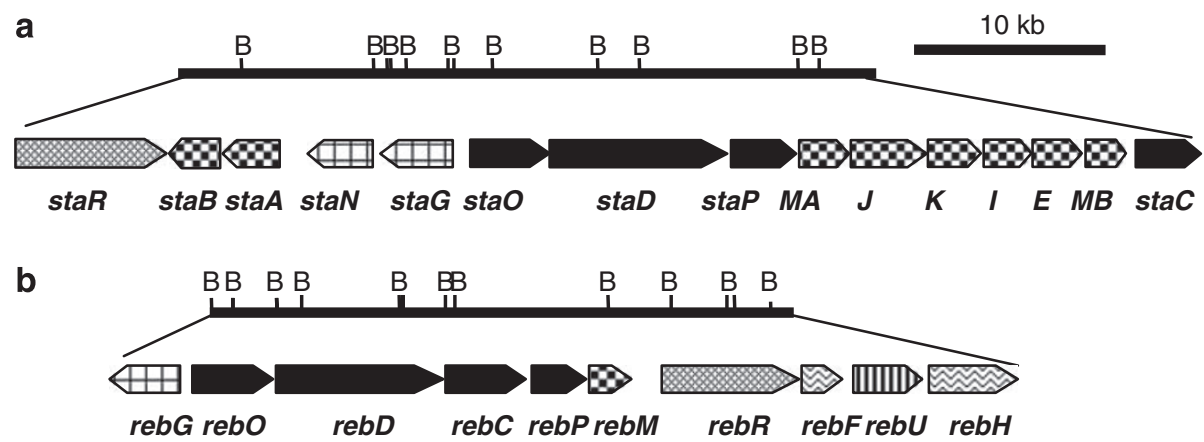

Indolocarabazole synthesis $\mathrm{BD}$ Deoxysugar synthesis
Chegulation

Figure 2 The gene cluster encoding biosynthesis of staurosporine and rebeccamycin. Restriction and organization chromosomal map of the sta locus (a) from Streptomyces TP-A0274 and reb locus (b) from Lechevalieria aerocolonigenes ATCC39243. 'B' indicates the BamHI sites (adapted from Onaka ${ }^{23}$ ).

of $3^{\prime}$-O-demethyl, $4^{\prime}$ - $N$-demethyl-staurosporine and staMB catalyzes $O$-methylation, which results in the formation of staurosporine.

In rebeccamycin biosynthesis, conversion of L-tryptophan to 7-chloro-L-tryptophan is the first step, the reaction being catalyzed by a two-component (halogenase rebH and flavin reductase rebF) enzyme. Subsequently, rebO and rebD catalyze 7-chloroindole-3pyruvic acid imine formation, and coupling of two imines yields 11,11-dichlorochromopyrrolic acid.

The genes involved in the main pathway of indolocarbazole structure formation in staurosporine and rebeccamycin showed striking similarity between $s t a O$, staD, staP, staC and $s t a G$, and $r e b O$, rebD, $r e b P$, rebC and $r e b G$, respectively. The formation of chromopyrrolic acid or 11,11-dichlorochromopyrrolic acid, key intermediates of indolocarbazole biosynthesis, is catalyzed by $s t a D$ or rebD. It is noteworthy that the staD family includes only two homologs, rebD and VioB, which are involved in violacein biosynthesis. The staD family is a new type of hemoprotein with a novel structure and function. ${ }^{25,26}$

$\mathrm{N}$-glycosidic bond formation between the $\mathrm{N}-12$ and $\mathrm{C}-\mathrm{1}^{\prime}$ positions is catalyzed by staG or rebG $N$-glycosyltransferases. rebG is the same gene that was cloned in 2000 by Ohuchi et al. as $n g t$, which can catalyze the $\mathrm{N}$-glycosylation of the indolocarbazole chromophore. In the staurosporine structure, there exists an additional, unusual $\mathrm{C}-\mathrm{N}$ bond between the $\mathrm{N}-13$ and $\mathrm{C}-6^{\prime}$ positions. Onaka et al. showed through gene disruption and bioconversion experiments that staN, a $\mathrm{P} 450$ homolog, is responsible for this unusual $\mathrm{C}-\mathrm{N}$ bond formation. StaN was the first example used to show that the P450 homolog is involved in N-glycosidic bond formation. Deletion of staG abolished glycosylation and led to accumulation of K252c, whereas deletion of staN resulted in the production of holyrine A. Salas et al. also showed the function of staN in $\mathrm{C}-\mathrm{N}$ bond formation by heterologous expression of the $\operatorname{staN}$ gene. ${ }^{27}$

\section{COMBINATORIAL BIOSYNTHESIS OF STAUROSPORINE ANALOGS}

Single-gene disruption studies and biochemical characterization of enzymes of staurosporine and rebeccamycin biosynthesis gene clusters have revealed the entire biosynthetic pathways of staurosporine and related indolocarbazole compounds. In addition, the entire sets of both staurosporine or rebeccamycin biosynthetic gene clusters were heterologously expressed in Streptomyces lividans by Onaka et al..$^{20}$ and in Streptomyces albus by Salas and collaborators, ${ }^{14,28}$ which resulted in the production of staurosporine or rebeccamycin in the surrogate hosts.

During and based on the above studies, new analogs of staurosporine and rebeccamycin have been identified as follows (Figure 4):

1. Intermediates of biosynthetic pathways

Although the majority of compounds identified as intermediates of the indolocarbazole biosynthetic pathways were previously isolated from culture broth as minor components, 11,11-dichlorochromopyrrolic acid was a new discovery that arised from use of an rebP disrupted mutant.

2. New analogs produced by heterologous gene expression

10-Hydroxy-staurosporine aglycone (10-hydroxy K252c) was produced in a staG-deficient strain by heterologous gene expression of ToxA gene, a tryptophan hydroxylase from Streptomyces mobaraense FERM BP-2785, which produces BE-13793C (1,11-dihydroxy, 7-oxostaurosporine aglycone). $\quad 3^{\prime}$-demethyl-3'-acetylstaurosporine was isolated from a staMA-blocked mutant.

3. Adaptability of enzymes in indolocarbazole biosynthetic pathways to unnatural substrates

Replacing sugar moiety: staG was shown to accept a variety of sugar derivatives. It has been used successfully for producing novel glycosylated indolocarbazoles consisting of L-rhamnose, L-olivose, L-digitoxose or D-olivose by Salas et al. In contrast to staG, no sugar derivative other than NDP-D-glucose has been reported as a substrate for rebG.

Tryptophan halogenase: $\mathrm{rebF} / \mathrm{rebH}$, tryptophan halogenase in rebeccamycin biosynthesis, accepts chloride and bromide ions. rebO could also use 1-methyl, 5-methyl and 5-fluoro-L-tryptophan as substrates.

4. Analogs with different oxidation states at the C-7 position

Many analogs with a different oxidation state at the C-7 position of the pyrrole ring in the indolocarbazole chromophore have been isolated. rebP and staP were functionally equivalent, and any of the cytochrome $P 450$ enzymes could be responsible for the decarboxylative oxidation of a chromopyrrolic acid intermediate into indolopyrrolocarbazole aglycone. A mixture of the three indolopyrrolocarbazole chromopyrrolic acids, which differ in their oxidation states at the C-7 position, were produced in the absence of staC or rebC. Interestingly, rebC and staC determine different oxidation states in the final product. Addition of $s t a C$ produced the single product K252c, whereas acryriaflavin A was produced by rebC. 
<smiles>N[C@@H](Cc1c[nH]c2ccccc12)C(=O)O</smiles>

L-Tryptophan<smiles>CC(C)CCC(=O)C(=O)O</smiles>

Indolepyruvic acid (IPA imine)<smiles>O=C(O)C1NC(C(O)O)C(C2CNC3CC4CCC3C4C2)C1C1CNC2CCCCC21</smiles>

Chromopyrrolic acid (CPA)

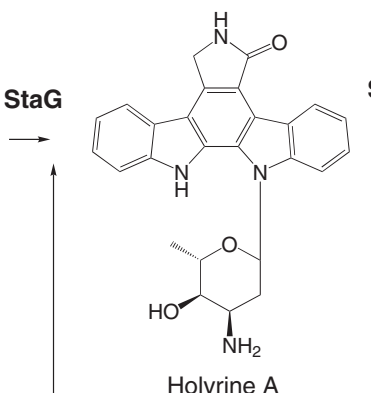

StaP StaC

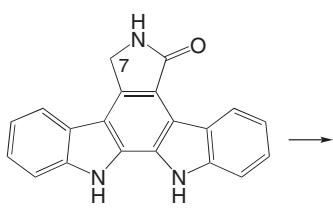

Staurosporine aglycon (K252c)

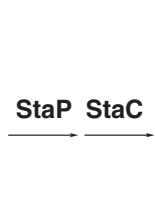

(N)

3'-O-demethyl-staurosporine

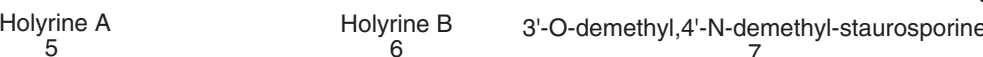

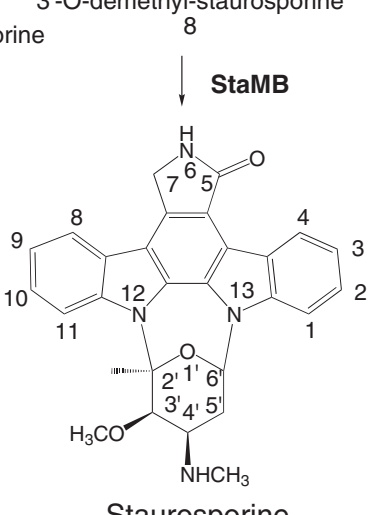

Staurosporine

b

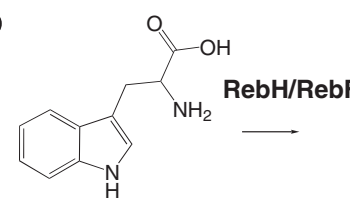
L-Tryptophan<smiles>COC(=O)c1cccc2c(C[C@@H](N)C(=O)O)c[nH]c12</smiles>

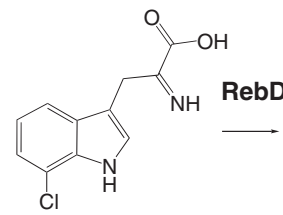
RebD<smiles>O=C(O)c1[nH]c(C(=O)O)c(-c2c[nH]c3c(Cl)cccc23)c1-c1c[nH]c2ccccc12</smiles>

RebP RebC

7-chloro-L-Tryptophan

7-Chloroindolepyruvic acid

11,11'-Dichlorochromopyrrolic acid<smiles>O=c1[nH]c(=O)c2c3c([nH]c4ccccc43)[nH]c3c(Cl)cccc3c1=2</smiles>

Rebeccamycin aglycon

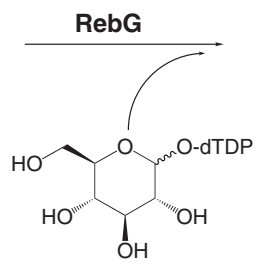

TDP-Glucose<smiles></smiles>

9

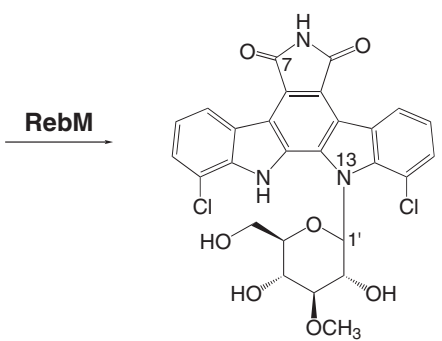

2 Rebeccamycin

Figure 3 The proposed overall biosynthetic pathways of staurosporine (a) and rebeccamycin (b) (modified from Onaka ${ }^{23}$ ).

\section{BIOLOGICAL ACTIVITIES}

Inhibition of mammalian protein kinases

In the mid-1980s, staurosporine and the related indolocarbazole K252 were shown to be potent inhibitors of protein kinases by Kyowa Hakko Co. (Kyowa Hakko Kirin Co. Ltd., Tokyo, Japan) ${ }^{29,30}$ Following the discovery that staurosporine was a nanomolar inhibitor of protein serine/ threonine kinases such as protein kinase $\mathrm{C}$ and also protein tyrosine kinase $\mathrm{v}$-src ${ }^{31}$ (Figure 5), many laboratories and pharmaceutical compa- nies sought selective protein kinase inhibitors by chemical synthesis or screening of new natural products. 7-Hydroxystaurosporine (UCN-01) was identified during microbial screening for selective protein kinase $\mathrm{C}$ inhibitor. ${ }^{32}$ UCN-01 showed antitumor activity in mouse tumor models and thus entered clinical studies. ${ }^{33}$

However, it was difficult to screen selective inhibitors against human protein kinases in the mid-1980s until bacculovirus expression systems for large-scale production of human proteins were developed 


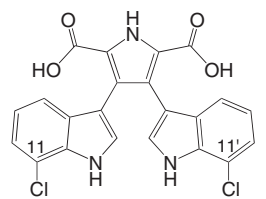

11,11'-Dichlorochromopyrrolic acid

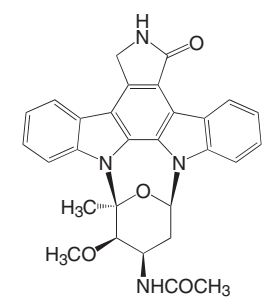

4'-N-demethyl-4-N'-acetyl-staurosporine

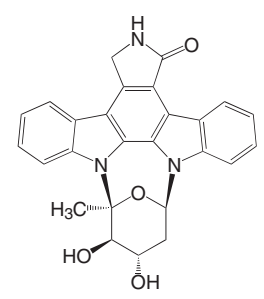

12,13-N,N-(L-olivopyranosyl)-K-252c<smiles>C[C@]12OC(C[C@@H](O)C1O)n1c3ccccc3c3c4c(c5c6ccccc6n2c5c31)CNC4=O</smiles>

12,13-N,N-(L-digitoxopyranosyl)-K-252c

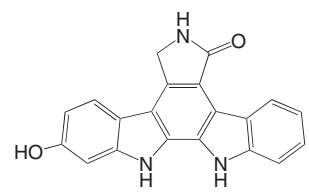

10-Hydroxy-K-252c

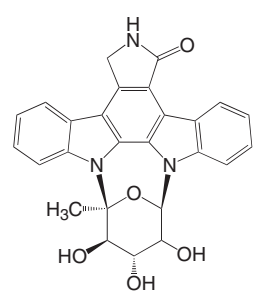

12,13-N,N-(L-rhamnopyranosyl)-K-252c

13-N-(L-olivopyranosyl)-K-252c

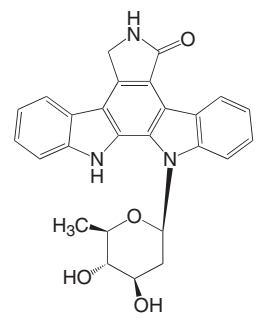

13-N-(D-olivopyranosyl)-K-252c

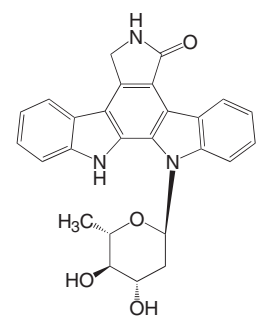

Figure 4 Chemical structures of new indolocarbazoles produced by combinatorial biosynthesis.

in the late 1980s. Researchers in Ciba-Geigy (Novartis International AG, Basel, Switzerland) started a protein kinase project in the mid1980s and, in the early 1990s, a synthetic inhibitor named CGP5714B (later named imatinib, structure in Figure 6) was discovered. In 1996, CGP5714B was shown to be a potent and selective inhibitor in colony formation assay against tumor cells from chronic myelogenous leukemia in which Bcr-Abl tyrosine kinase is activated. ${ }^{34}$ Imatinib (Gleevec) entered human clinical trials on chronic myelogenous leukemia in 1998 and was approved for use in 2001 in the USA.

Following the success of imatinib, EGF receptor tyrosine kinase inhibitors such as gefitinib (Iressa) and erlotinib (Tarceva) were approved for the treatment of lung cancer. Consequently, protein kinases have become the second most important drug targets after G-protein-coupled receptors. ${ }^{7}$

Staurosporine-related structures (for example, indolocarbazole aglycone, diindolylmaleimides and dianilinophthalamides) have been derivatized and developed into selective inhibitors of pharmacologically interesting targets. The structure of several representative compounds evaluated in clinical studies on cancer, such as midostaurin (CGP41251), lestaurtinib (CEP-701, KT-5555) and enzaistaurin (LY317815), are shown in Figure 6. ${ }^{7,14,35,36}$

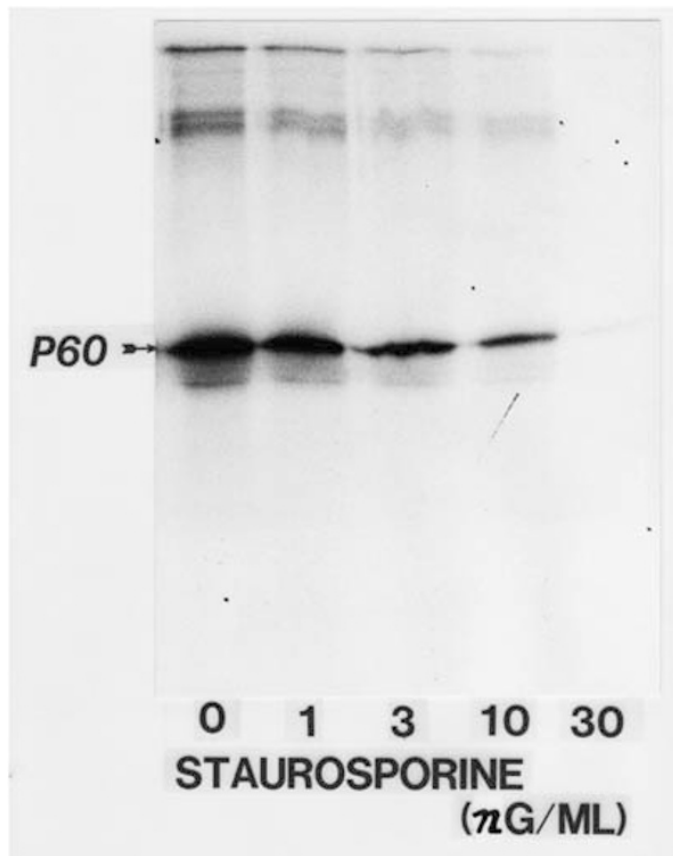

Figure 5 Inhibition of tyrosine-specific protein kinase activity of p60 ${ }^{v-s r c}$ by staurosporine. $\mathrm{p} 60^{\mathrm{v}-\mathrm{src}}$ was immunoprecipitated from the lysates of chicken embryo fibroblasts infected with Raus sarcoma virus by anti-p60 serum. The immunoprecipitates were incubated with kinase assay buffer $820 \mathrm{~mm}$ Tris- $\mathrm{HCl}(\mathrm{pH} 7.2), 5 \mathrm{~mm} \mathrm{MgCl}_{2}$ and $10 \mu \mathrm{Ci}\left[\gamma_{-}{ }^{32} \mathrm{P}\right]$ ATP for $30 \mathrm{~min}$ at $20^{\circ} \mathrm{C}$. Staurosporine was dissolved, diluted in dimethyl sulfoxide (DMSO) and added to the assay buffer at the concentrations indicated. The reaction products were analyzed on SDS-10\% acrylamide gel. The dried gel was exposed to X-ray film for 90 min (adapted from Nakano et al. ${ }^{31}$ ).

In addition to antitumor activity, staurosporine and K252a derivatives possess many other useful pharmacological properties. The $\gamma$-lactone form of staurosporine was shown to have antiplatelet aggregation activity, although it has more than 100-fold lower activity against protein kinase $\mathrm{C}$ and smooth muscle contraction. ${ }^{37} \mathrm{~A}$ series of 3,9-disubstituted K252a derivatives have been synthesized and evaluated for neurotrophic activity. KT-7515 (CEP1347), an ethylthiomethyl derivative of $\mathrm{K} 252 \mathrm{a}$, showed reduced kinase inhibitory properties for trk A, PKC and PKA while enhancing neurotrophic activity. ${ }^{38}$

Staurosporine was shown to inhibit many protein serine-threonine kinases and tyrosine kinases, and has been used as the reference compound for various protein kinase assays. In 2002, based on human genome sequence data, 518 protein kinase genes were identified (about $1.7 \%$ of the total number of human structure genes). ${ }^{39}$ Figure 7 shows the $\mathrm{IC}_{50}$ values of staurosporine against 235 protein kinases assayed using kinases produced by bacculovirus expression systems. ${ }^{40}$ Figure 8 shows the inhibitory profiles of imatinib and gefitiniib, determined under the same assay conditions. ${ }^{40}$ The inhibitory profile of imatinib is not directly linked to the position of protein kinases on the evolutionary dendrogram; it exhibits comparable efficiencies at inhibiting PDGFR and KIT, which are more divergent from the Abl tyrosine kinase than the SRC subfamily, while also exhibiting low inhibition potency with regard to the tyrosine kinase activity of the SRC family.

The crystal structures of staurosporine and protein kinases, including PKC isozymes, cyclin-dependent kinases and EGF receptor tyrosine kinase, showed that several binding sites of staurosporine overlap with those of ATP. ${ }^{41}$ Further, comparison of the crystal structures of staurosporine and UCN-01 in complex with the kinase domain of CHK1 (cell cycle checkpoint kinase-1) and PDK1 


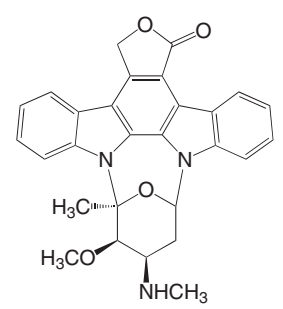

Staurosporine latone form

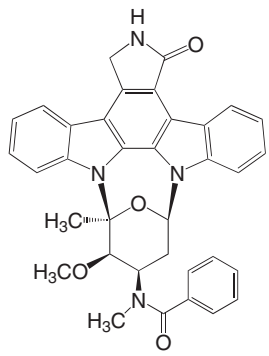

Midostaurin (CGP41251)<smiles>CN(C)CC1CCn2c3ccccc3c3c4c(c5c6ccccc6n2c5c43)C(=O)NC1=O</smiles>

Ruboxistaurin (LY333531)<smiles></smiles>

$\mathrm{KT}-6528\left(\mathrm{R}=\mathrm{CH}_{2} \mathrm{OH}\right)$ $\mathrm{KT}-6661\left(\mathrm{R}=\mathrm{CO}_{2} \mathrm{CH}_{3}\right)$<smiles>N#CCCn1c2ccccc2c2c3c(c4c5ccccc5[nH]c4c21)C(=O)NC3</smiles>

Enzaistaurin (LY317815)

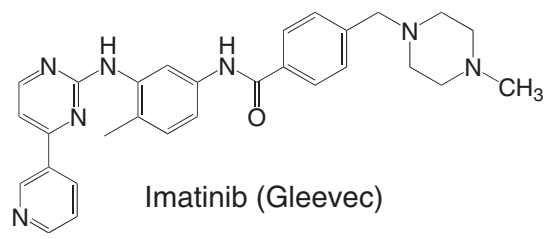

Figure 6 Chemical structures of some synthetic indolocarbazole derivatives and mimics that stimulate interest in drug discovery targeting protein tyrosine kinases and topoisomerases.

(3-phosphoinositide-dependent protein kinase-1) showed that the 7-hydroxy group of UCN-01 generates additional hydrogen-bonding interactions with active site residues, directly to threonine or serine and indirectly through an ordered water ${ }^{42}$ (Figure 9).
In 2004, the crystal structure of Gleevec bound to Syk (Spleen tyrosine kinase) revealed that imatinib binds Syk in a novel, compact cis conformation that differs from the binding mode observed with unphosphorylated Abl, the imatinib-sensitive form of Abl. The cis conformation of imatinib bound to Syk closely resembles the structure of staurosporine bound to Syk. The pyridine, pyrimidine and benzene rings of imatinib closely correspond with all the three coplanar arms of staurosporine $^{43}$ (Figure 10).

\section{Anti-malarial and anti-trypanosomal drug targeting protein kinases in protozoa}

New drugs and molecular targets are needed against human parasitic protozoa, such as Plasmodium falciparum and Trypanosoma brucei, due to the increased resistance to existing drugs. ${ }^{44}$ The clinical success of protein kinase inhibitors against various human diseases stimulated a search for protein kinases in human parasite protozoa. Prior to the genome sequence analysis of the human malarial parasite $P$. falciparum, several plasmodial protein kinases were identified through homology with mammalian protein kinases. The three-dimensional structure of PfPK5, a cyclin nuclear division cycle-dependent kinase (CDK) in $P$. falciparum, has been identified. Staurosporine inhibits PfPK5 with an $\mathrm{IC}_{50} 1000 \mathrm{~nm}$ less potent than that of the CDK2 inhibitors NU6102 (215 nM) and purvalanol B (130 nM). ${ }^{45}$

In 2004, completion of the whole genome sequence of $P$. falciparum revealed profound divergences between kinomes of the parasite and those of its host. About one-third of the 85 Plasmodium protein kinases are 'orphans,' which do not cluster with any of the protein kinase families established from any mammalian or yeast kinomes. These may turn out to represent very attractive targets for novel, parasite-specific protein kinase inhibitors. In addition, homology of ortholog genes showed $40-60 \%$ similarity between the host and the parasite; hence, it will be highly beneficial to identify protein kinase inhibitors with 'parasite versus host' selectivity targeting protein kinases that are essential for parasite development. ${ }^{46}$

In 2005, the sequenced genomes of three human trypanosomatid protozoa, Leishmania major, T. brucei and Trypanosoma Cruzi, showed that they encode 179, 156 and 171 eukaryotic protein kinases, respectively (about one-third of the human complement). ${ }^{47}$

\section{Activity against microbial and viral protein kinases}

Staurosporine was shown to have antifungal activity, but to lack significant antibacterial activity. However, in the 1990s, many protein Ser/Thr kinases were identified in prokaryotes and viruses, including several pathogenic organisms. Reports suggested that protein kinases in pathogenic mycobacteria could be valuable new therapeutic targets for antituberculosis drug discovery. ${ }^{48}$ In 2007, K252a, K252b and staurosporine were shown to inhibit a mycobacterial protein kinase PknB with $\mathrm{IC}_{50}$ values of 96, 106 and $600 \mathrm{~nm}$, respectively. The PknB gene is strictly conserved in all known mycobacterial genomes and some related actinomycetes. $\mathrm{PknB}$ is a receptor-like transmembrane protein, with an extracellular signal sensor domain and an intracellular kinase domain that shares similarity with eukaryotic protein kinases. Staurosporine and K252a were found to inhibit the growth of Mycobacterium tuberculosis $\mathrm{H} 37 \mathrm{Rv}$ at $5-50 \mu \mathrm{M}$, whereas $\mathrm{K} 252 \mathrm{~b}$ failed to inhibit the growth. ${ }^{49}$

The protein kinases were considered to be evolutionarily segregated into eukaryotic serine/threonine/tyrosine kinases and prokaryotic histidine kinase. However, genome sequences of bacteria revealed an abundance of serine/threonine/tyrosine protein kinases (for example, in pathogenic strains; 13 in M. tuberculosis and 5 in Corynebacterium diphtheriae $^{50}$ In addition, phosphoproteome analysis using high- 

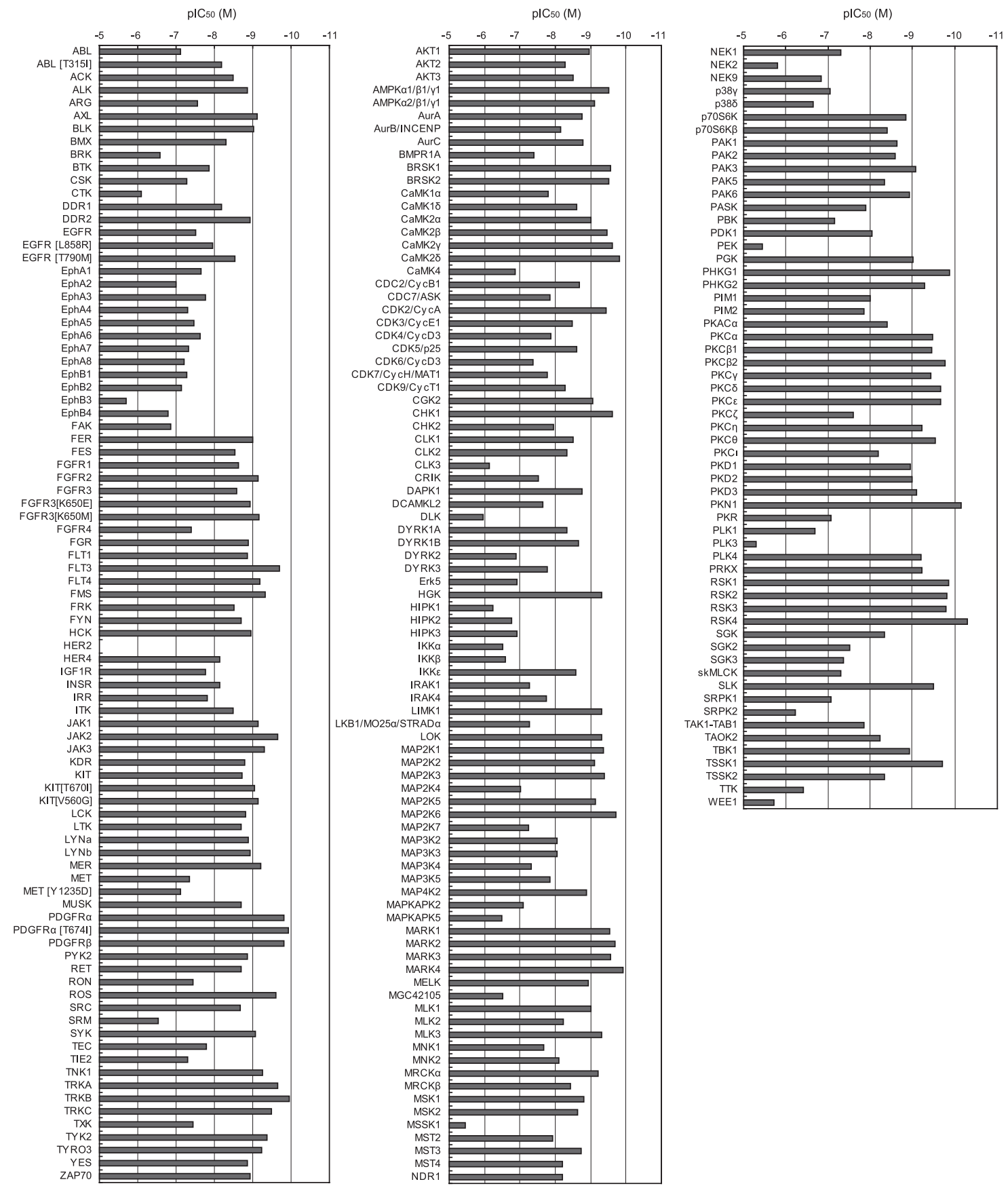

Figure $7 \mathrm{IC}_{50}$ of staurosporine against 235 protein kinases (by Carna Bioscience Inc., Kobe, Japan). Inhibition profiles were assayed by mobility shift assay using kinases produced as N-terminal glutathione $S$-transferase (GST) fusion protein by bacculovirus expression systems and purified by glutathione sepharose chromatography. The kinase reaction takes place in a 384-well plate. A capillary sipper transfers the sample into the microchip, which serves as a separation device for the substrate, and the phosphorylated product is detected through laser-induced fluorescence. The substrate design and peak separation condition of the substrate and products are critical and were optimized for each kinase. Assay conditions for each kinase are published in a kinase profiling book by Carna Bioscience Inc.: http://www.carnabio.com/output/pdf/ProfilingProfilingBook_ja.pdf.

resolution mass spectrometry showed that the ratio of phosphotyrosine, phosphothreonine and phosphoserine is 10:20:70 in Bacillus subtilis, similar to the ratio of 2:12:86 in human cells as determined by the same method. ${ }^{51}$

Indolocarbazoles also possess antiviral properties, including activity against the human immunodeficiency virus, cytomegalovirus and Epstein-Barr virus. ${ }^{52-54}$ Several indolocarbazoles inhibit the protein kinase activity of pUL97, encoded by human cytomegalovirus, NGIC-
I being the most effective, exhibiting an $\mathrm{IC}_{50}$ of $42 \mathrm{~nm}$, and an $\mathrm{IC}_{50}$ of $38 \mathrm{~nm}$ in an antiviral assay using green fluorescent protein expressing recombinant human cytomegalovirus.

\section{Action on topoisomerases}

DNA topoisomerases have been shown to be important targets for antitumor drugs and antibacterial agents. Both antitumor drugs in eukaryotes and quinolone antibiotics in prokaryotes stabilize 

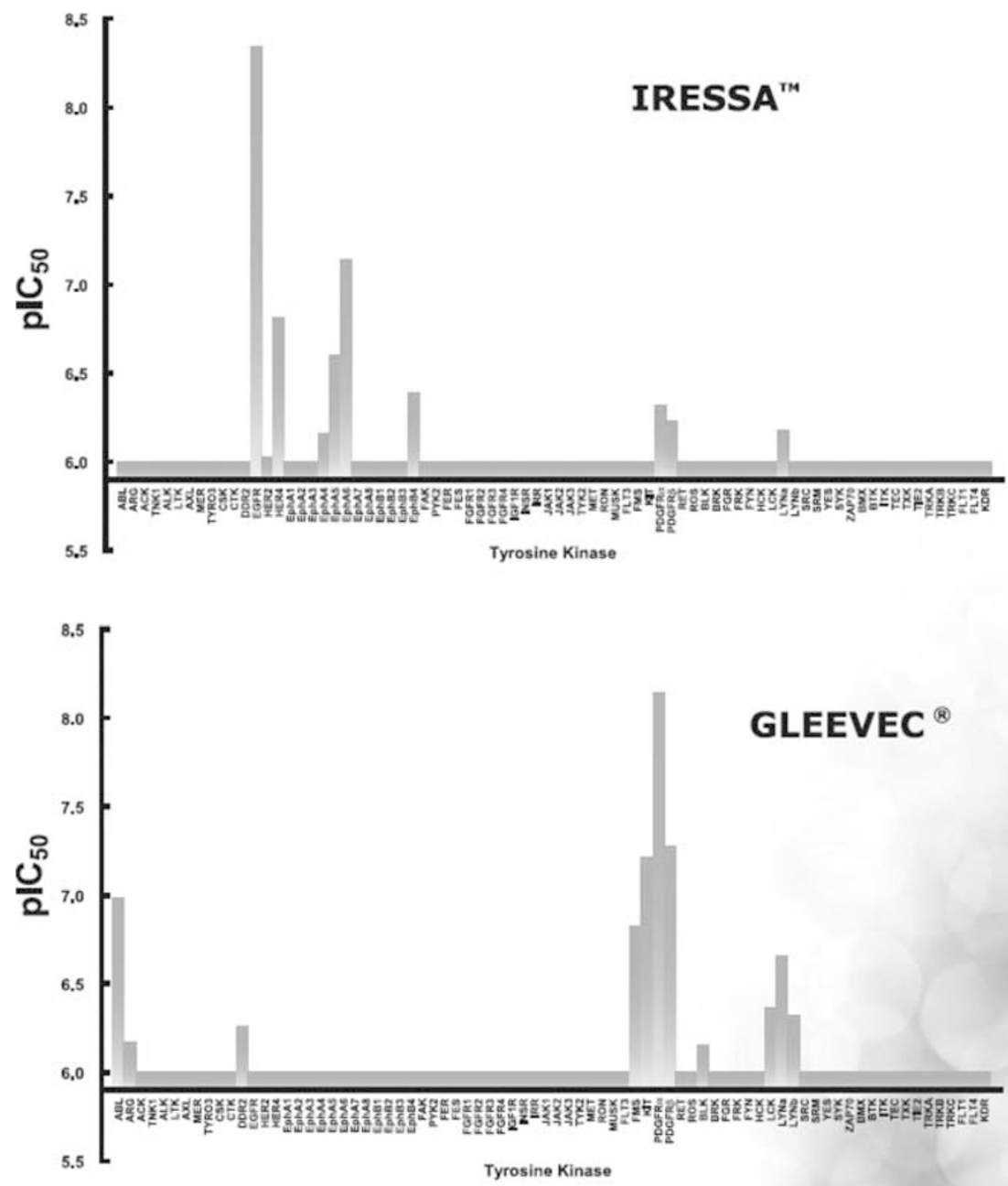

Figure 8 Kinase profiling results of imatinib (Gleevec) and gefitinib (Iressa) (by Carna BioScience). ${ }^{40}$ Adapted from a publication in 'Screening-Trends in Drug Discovery' (journal issued by GIT Verlag GmbH \& Co. KG).

DNA-cleavable complexes with the topoisomerases of target cells. In 1992, Yamashita et al. showed that K252 derivatives, such as KT-6006, KT-6528 and KT-6661 (structures in Figure 6) induce a DNAcleavable complex with mammalian topoisomerase I. ${ }^{8}$ These semisynthetic derivatives of $\mathrm{K} 252 \mathrm{a}$ were potent inducers of DNA-cleavable complexes with topoisomerase I, whereas rebeccamycin was a weak inducer in the same assay (Figure 11).

Many antitumor indolocarbazoles targeting topoisomerase have since been synthesized. Indolocarbazoles are now the most advanced non-camptothecin topoisomerase I inhibitors in clinical development. ${ }^{55}$ In 1996, topoisomerase I was shown to have an intrinsic protein kinase activity (Topo I kinase). Topo I kinase is required for phosphorylation of the SR protein that functions in ESE (exonic splicing enhancer)-dependent splicing. ${ }^{10}$ Human topoisomerase I exhibits at least two different conformations: (1) as drug targets in the form of a complex with substrates of the kinase reaction and (2) a topoisomerase I-DNA complex. Based on the common structure of ternary trapping by small molecules of the intermediate protein complex, such as a topoisomerase I-DNA covalent complex, Pommier et al. proposed the 'interfacial inhibitor paradigm.' This paradigm described for topoisomerase I inhibitors can be generalized to cover a variety of natural products that trap macromolecular complexes that undergo catalytic conformational changes providing hotspots for drug binding. ${ }^{56}$ Staurosporine and related indolocarbazoles represent natural products that can trap several of these interfacial macromolecular complexes.

\section{CONCLUSION}

We have reviewed several key components of the chemistry and biology of natural indolocarbazole products elucidated during the three decades since the discovery of staurosporine in 1977. Over the same timeframe, remarkable progress has been made in molecular and cellular biology, such as molecular target identification of human diseases, genome-based research on the human genome and analysis of crystal structures of drug-protein complexes using human proteins produced by bacculovirus-insect cell expression systems. Staurosporine and related natural indolocarbazole products have attracted not only scientists in drug discovery and development but also chemists and biologists engaged in biosynthetic gene discovery, combinatorial biosynthesis and cell biology. Owing to space constraints, we have not been able to describe an important activity of staurosporine, namely the induction of cell death (apoptosis). To our surprise, a search for 'staurosporine' plus 'apoptosis' on 'Google scholar' resulted in 18300 hits. Staurosporine shows an extremely strong cytotoxic activity in some cases (4 pM, Hela-S3, $72 \mathrm{~h}$ exposure) and induces apoptosis. Several tumor cell lines are completely resistant to different anticancer 
drugs, but remain sensitive to staurosporine-induced apoptosis. ${ }^{57}$ Further research on the use of staurosporine derivatives in anticancer therapy is clearly necessary, and the molecular mechanism of staurosporine-induced apoptosis remains to be determined. ${ }^{58}$

In the mid-2000s, nearly 30 years after the discovery of staurosporine, it was revealed that $\mathrm{ABCG} 2$, an $\mathrm{ABC}$ transporter with importance in cancer drug resistance, oral drug absorption and stem cell biology, is a promising new target for indolocarbazoles such as UCN-01 and bisindolylmaleimide. ${ }^{59,60}$ Consequently, we predict that a library of compounds derived from staurosporine and related indolocarbazoles will have huge potential for inhibition of new targets with ATP-binding domains, in addition to mammalian protein kinases, topoisomerases and $\mathrm{ABC}$ transporters.

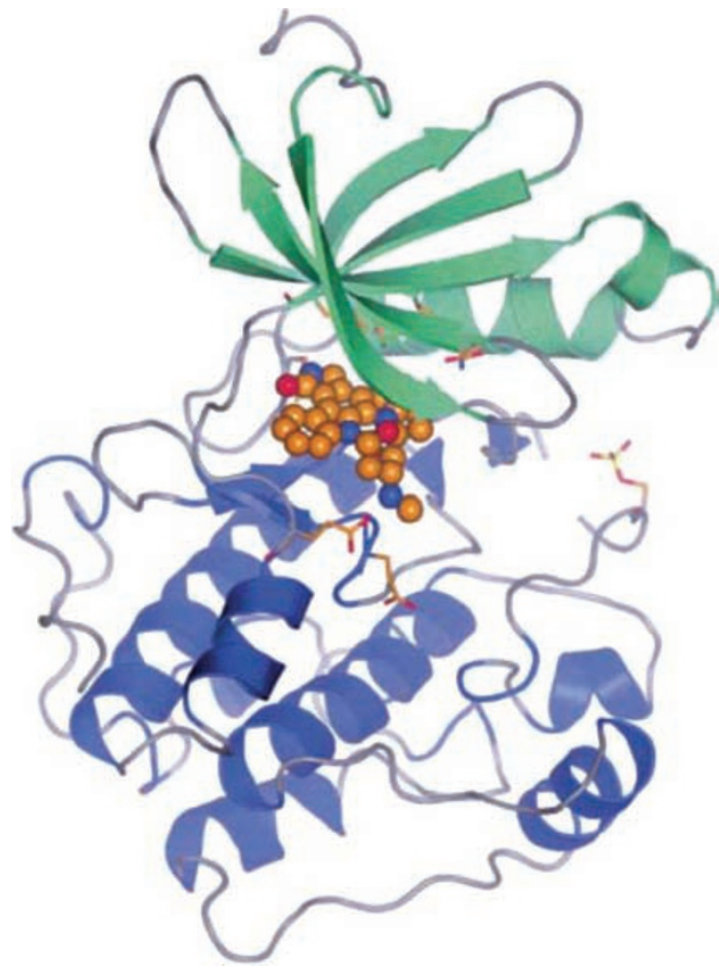

Figure 9 Overview of PDK1 kinase domain bound to staurosporine. The two lobes (in green ( $\mathrm{N}$-terminal lobe) and blue (C-terminal lobe)) of the kinase enclose the inhibitor molecule (shown by the orange spheres). The phosphorylated T-loop (shown as a stick representation with a yellow phosphorus atom and red oxygen atoms) lacks residues, due to disorder. Side chains interacting with the inhibitor molecule are shown as a stick representation with green carbon atoms (adapted from Komander et al. ${ }^{42}$ ). See online version for color figure.

Figure 11 Mammalian DNA topoisomerase I-mediated DNA cleavage activities of indolocarbazole derivatives. Methods: In DNA cleavage assay, $0.48 \mu \mathrm{g}$ of supercoiled pBR322 DNA was incubated with $100 \mathrm{U}$ of topoisomerase I in the presence of drugs (lanes $\mathrm{c}-\mathrm{t}$ ) followed by SDS/proteinase $\mathrm{K}$ treatment, and was then analyzed on an agarose gel containing $0.5 \mu \mathrm{gml}^{-1}$ ethidium bromide. Lane a, CCC-DNA control; lane b, no drug; lanes c-e, K252a; lanes f-h, KT6006; lanes i-k, KT6661; lanes I-n, KT6528; lanes o-q, rebeccamycin; lanes $\mathrm{r}-\mathrm{t}$, camptothecin. Drug concentrations were (lanes c, $\mathrm{f}, \mathrm{i}, \mathrm{I}, \mathrm{o}$ and $\mathrm{r}$ ) 0.5 , (lanes d, g, j, m, p and s) 5 and (lanes e, h, k, n, q and t) $50 \mu \mathrm{m}$. Results: KT6006 induced topoisomerase I-mediated DNA cleavage in a dose-dependent manner at drug concentrations up to $50 \mu \mathrm{m}$, whereas DNA cleavage induced by KT6528 was saturated at $5 \mu \mathrm{m}$. The maximal amount of nicked DNA produced by KT6006 was more than $50 \%$ of substrate DNA, which was comparable to that of camptothecin (adapted from Yamashita et al. ${ }^{8}$ ).
Clearly, staurosporine and related natural products have a supreme ternary structure helping in their interaction with several important drug target proteins. They represent a splendid and highly adaptable
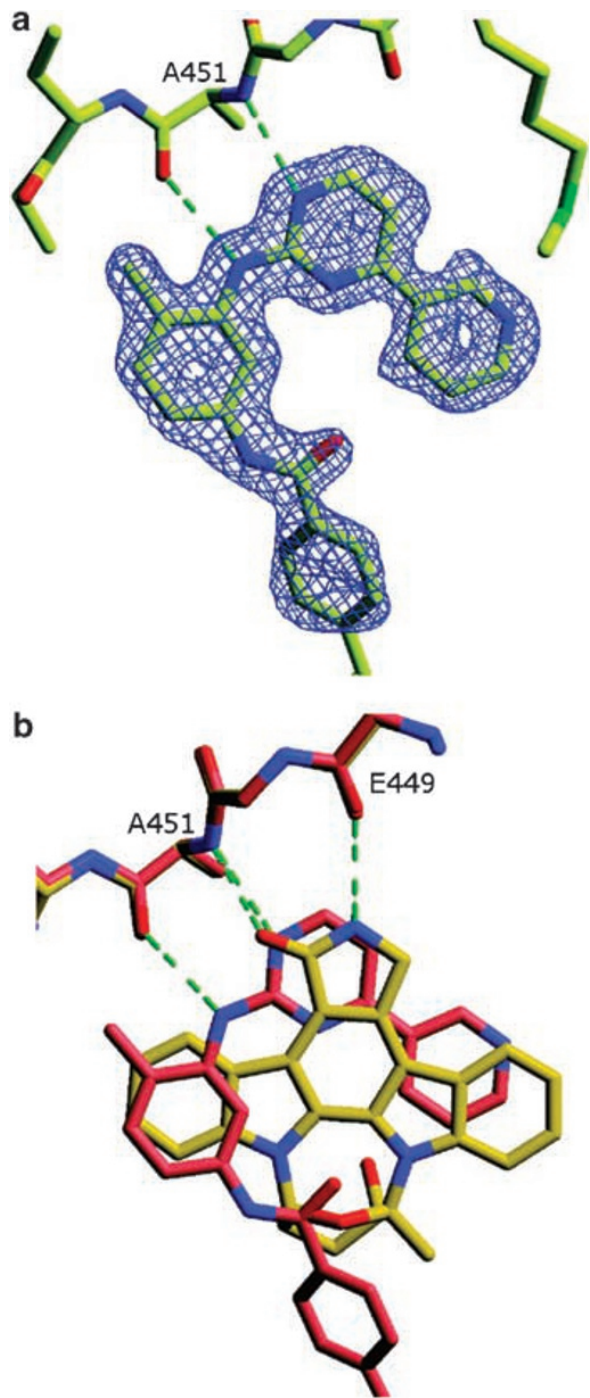

Figure 10 Structure of imatinib (Gleevec) bound to Syk (spleen tyrosine kinase) bearing a striking resemblance to the structure of staurosporine. (a) Binding of imatinib to Syk. Ligand difference in electron density. (b) Binding of imatinib and staurosporine to Syk. Imatinib (yellow) binding to Syk in the compact cis conformation mimics the structure and binding mode of staurosporine (pink). The key residues are labeled and hydrogen bonds are denoted with dashed lines (adapted from Atwell et al. ${ }^{43}$ ). See online version for color figure.

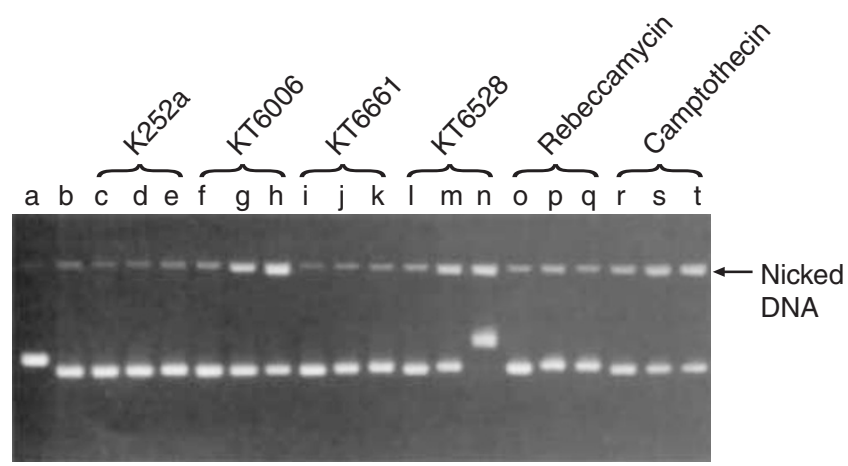


gift from nature, which can be manipulated by human endeavor to bestow immeasurable benefit for human health.

\section{ACKNOWLEDGEMENTS}

We thank Hiroyasu Onaka (Toyama Prefectural University), Toshiaki Sunazuka and Yoko Takahashi (Kitasato Institute), Chikara Murakata, Yoshinori Yamashita and Isami Takahasi (Kyowa Hakko Kirin Co. Ltd.) for their helpful discussions. We apologize to the many scientists whose important discoveries could not be included or referenced in this article because of space restrictions.

1 Ōmura, S. Philosophy of new drug discovery. Microbiol. Rev. 50, 259-279 (1986).

2 Newman, D., Cragg, G. M. \& Snader, K. M. The influence of natural products upon drug discovery. Nat. Prod. Rep. 17, 215-234 (2000).

3 Clardy, J. \& Walsh, C. Lessons from natural molecules. Nature 432, 829-837 (2004).

4 Drews, J. Drug discovery: a historical perspective. Science 287, 1960-1964 (2000).

5 Ōmura, S., Sasaki, Y., Iwai, Y. \& Takeshima, H. Staurosporine, a potentially important gift from a microorganism. J. Antibiot. 48, 535-548 (1995).

6 Tamaoki, T. \& Nakano, H. Potent and specific inhibitors of protein kinase C from microbial origin. Biotechnology 8, 732-735 (1990).

7 Cohen, P. Protein kinases - the major drug targets of the twenty-first century. Nat. Rev. Drug Discov. 1, 309-315 (2002).

8 Yamashita, Y. et al. Induction of mammalian DNA topoisomerase I mediated DNA cleavage by antitumor indolocarbazole derivatives. Biochemistry 31, 12069-12075 (1992).

9 Wang, J. C. Cellular roles of DNA topoisomerases: a molecular perspective. Nat. Rev. Mol. Cell. Biol. 3, 430-440 (2002).

10 Rossi, F. et al. Specific phosphorylation of SR proteins by mammalian DNA topoisomerase I. Nature 381, 80-82 (1996)

11 Ōmura, S. et al. New alkaloid AM-2282 of Streptomyces origin: taxonomy, fermentation, isolation and preliminary characterization. J. Antibiot. 30, 275-282 (1977).

12 Takahashi, Y., Shinose, M., Seino, A., Iwai, Y. \& Ōmura, S. Transfer of staurosporineproducing strain Streptomyces staurosporeus AM-2282 to the genus Saccharothrix as Saccharothrix aerocolonigenes (Labeda 1986) subsp. staurosporeus subsp. nov. Actinomycetologica 9, 19-26 (1995).

13 Xie, Q. et al. Description of Lentzea flaviverrucosa sp. nov. and transfer of the type strain of Saccharothrix aerocolonigenes subsp* staurosporea to Lentzea albida. Int. J. Syst. Evol. Microbiol. 52, 1815-1820 (2002).

14 Sanchez, C., Mendez, C. \& Salas, J. A. Indolocarbazole natural products: occurrence, biosynthesis, and biological activity. Nat. Prod. Rep. 23, 1007-1045 (2006).

15 Takahashi, I., Asano, K., Kawamoto, I., Tamaoki, T. \& Nakano, H. UCN-01 and UCN02 , new selective inhibitors of protein kinase C. I. Screening, producing organism and fermentation. J. Antibiot. 42, 564-570 (1989).

16 Hong, S. K., Matsumoto, A., Horinouchi, S. \& Beppu, T. Effects of protein kinase inhibitors on in vitro protein phosphorylation and cellular differentiation of Streptomyces griseus. Mol. Genet. Genomics 236, 347-354 (1993).

17 Ōmura, S. et al. Genome sequence of an industrial microorganism, Streptomyces avermitilis: deducing the ability for producing secondary metabolites. Proc. Natl. Acad. Sci. USA 98, 12215-12220 (2001).

18 Bentley, S. D. et al. Complete genome sequence of the model actinomycete Streptomyces coelicolor A3(2). Nature 417, 141-147 (2002).

19 Ohuchi, T. et al. Cloning and expression of a gene encoding $\mathrm{N}$-glycosyltransferase (ngt) from Saccarothrix aerocolonigenes ATCC39243. J. Antibiot. 53, 393-403 (2000).

20 Onaka, H., Taniguchi, S., Igarashi, Y. \& Furumai, T. Cloning of the staurosporine biosynthetic gene cluster from Streptomyces sp. TP-A0274 and its heterologous expression in Streptomyces lividans. J. Antibiot. 55, 1063-1071 (2002).

21 Onaka, H., Taniguchi, S., Igarashi, Y. \& Furumai, T. Characterization of the biosynthetic gene cluster of rebeccamycin from Lechevalieria aerocolonigenes ATCC 39243. Biosci. Biotechnol. Biochem. 67, 127-138 (2003).

22 Sanchez, C. et al. The biosynthetic gene cluster for the antitumor rebeccamycin; characterization and generation of indolocarbazole derivatives. Chem. Biol. 9, 519-531 (2002).

23 Onaka, H. Biosynthesis of heterocyclic antibiotics in actinomycetes and an approach to synthesize the natural compounds. Actinomycetologica 20, 62-71 (2006).

24 Makino, M. et al. Crystal structures and catalytic mechanism of cytochrome $P 450$ StaP that produces the indolocarbazole skeleton. Proc. Natl. Acad. Sci. USA 104, 11591-11596 (2007).

25 Howard-Jones, A. R. \& Walsh, C. T. Enzymatic generation of chromopyrrolic acid scaffold of rebeccamycin by the tandem action of RebO and RebD. Biochemistry 44, 15652-15663 (2005).

26 Asamizu, S., Kato, Y., Igarashi, Y., Furumai, T. \& Onaka, H. Direct formation of chromopyrrolic acid from indole-3-pyruvic acid by StaD* a novel hemoprotein in indolocarbazole biosynthesis. Tetrahedron Lett. 47, 473-475 (2006).

27 Salas, A. P. et al. Deciphering the late steps in the biosynthesis of anti-tumor indolocarbazole staurosporine: sugar donor substrate flexibility of the StaG glycosyltransferase. Mol. Microbiol. 58, 17-27 (2005).
28 Sanchez, C. et al. Combinatorial biosynthesis of antitumor indolocarbazole compounds. Proc. Natl. Acad. Sci. USA 102, 461-466 (2005).

29 Tamaoki, T. et al. Staurosporine, a potent inhibitor of phospholipid/Ca ${ }^{++}$dependent protein kinase. Biochem. Biophys. Res. Commun. 135, 397-402 (1986).

30 Kase, H., Iwahashi, K. \& Matsuda, Y. K-252a, a potent inhibitor of protein kinase C from microbial origin. J. Antibiot. 39, 1059-1065 (1986).

31 Nakano, H. et al. Staurosporine inhibits tyrosine-specific protein kinase activity of Rous sarcoma virus transforming protein p60. J. Antibiot. 40, 706-708 (1987).

32 Takahashi, I., Kobayashi, E., Asano, K., Yoshida, M. \& Nakano, H. UCN-01, a selective inhibitor of protein kinase $\mathrm{C}$ with antitumor activity from Streptomyces. J. Antibiot. 40, 1782-1784 (1987).

33 Akinaga, S., Gomi, K., Morimoto, M., Tamaoki, T. \& Okabe, M. Antitumor activity of UCN-01, a selective inhibitor of protein kinase $\mathrm{C}$, in murine and human tumor models. Cancer Res. 51, 4888-4892 (1991).

34 Druker, B. J. et al. Effects of a selective inhibitor of Abl tyrosine kinase on the growth of Bcr-Abl positive cells. Nat. Med. 2, 561-566 (1996).

35 Weisberg, E. et al. Inhibition of mutant FLT3 receptors in leukemia cells by the small molecule tyrosine kinase inhibitor PKC412. Cancer Cell 1, 433-443 (2002).

36 Engel, G. L., Farid, N. A., Faul, M. M., Richardson, L. A. \& Winneroski, L. L. Salt form selection and characterization of LY 333531 mesylate monohydrate. Int. J. Pharm. 198, 239-247 (2000).

37 Yamada, R. et al. Synthesis and activity of staurosporine analogs with a lactone functionality. Bioorg. Med. Chem. Lett. 6, 1893-1896 (1996).

38 Kaneko, M. et al. Neurotrophic 3,9-bis[(alkylthio)methyl]- and -bis(alkoxymethyl)-K252a derivatives. J. Med. Chem. 40, 1863-1869 (1997).

39 Manning, G., Whyte, D. B., Martinez, R., Hunter, T. \& Sudarsanam, S. The protein kinase complement of the human genome. Science 298, 1912-1934 (2002).

40 Kinase Profiling Book by Carna Bioscience Inc.. http://www.carnabio.com/output/pdf/ ProfilingProfilingBook_ja.pdf

41 Toledo, L. M. \& Lydon, N. B. Structures of staurosporine bound to CDK2 and CAPK-new tools for structure-based design of protein kinase inhibitors. Structure $\mathbf{5}$, 1551-1556 (1997).

42 Komander, D. et al. Structural basis for UCN-01 (7-hydroxystaurosporine) specificity and PDK1 (3-phosphoinositide-dependent protein kinase-1) inhibition. Biochem. J. 375, 255-262 (2003).

43 Atwell, S. et al. A novel mode of Gleevec binding is revealed by the structure of spleen tyrosine kinase. J. Biol. Chem. 279, 55827-55832 (2004).

44 Ridley, R. G. Medical need, scientific opportunity and the drive for antimalarial drugs. Nature 415, 686-693 (2002).

45 Holton, S. et al. Structures of $P$. falciparum PfPK5 test the CDK regulation paradigm and suggest mechanisms of small molecule inhibition. Structure 11, 1329-1337 (2003).

46 Doerig, C. \& Meijer, L. Antimalarial drug discovery: targeting protein kinases. Expert Opin. Ther. Targets 1, 279-290 (2007).

47 Parsons, M., Worthey, E. A., Ward, P. N. \& Mottram, J. C. Comparative analysis of the kinomes of three pathogenic trypanosomatids; Leishmaniamajor, Trypanosoma brucei and Trypanosoma cruzi. BMC Genomics 6, 127 (2005).

48 Kang, C. M. et al. The Mycobacterium tuberculosis serine/threonine kinases PknA and PknB: substrate identification and regulation of cell shape. Genes Dev. 19, 16921704 (2005).

49 Fernandez, $P$. et al. The Ser/Thr protein kinase $\mathrm{PknB}$ is essential for sustaining mycobacterial growth. J. Bacteriol. 188, 7778-7784 (2006).

50 Kenelly, P. J. Protein kinases and protein phosphatases in prokarytes: a genome perspective. FEMS Microbiol. Lett. 206, 1-8 (2002).

51 Macek, B. et al. The serine/threonine/tyrosine phosphoproteome of the model bacterium Bacillus subtilis. Mol. Cell. Proteomics 6, 697-707 (2007).

52 Marschall, M. et al. Direct targeting of human cytomegalovirus protein kinase pUL97 by kinase inhibitors is a novel principle for antiviral therapy. J Gen. Virol. 83, 1013-1023 (2002).

53 Gershburg, E., Hong, K. \& Pagano, J. S. Effects of maribavir and selected indolocarbazoles on Epstein-Barr virus protein kinase BGLF4 and on viral lytic replication. Antimicrob. Agents Chemother. 48, 1900-1903 (2004).

54 Gershburg, E. \& Pagano, J. S. Conserved herpes virus protein kinases. Biochim. Biophys. Acta 1784, 203-212 (2008).

55 Pommier, Y. Topoisomerase I inhibitors: camptothecins and beyond. Nat. Rev. Cancer 6, 789-802 (2006).

56 Marchand, C. M. et al. A novel norindenoisoquinoline structure reveals a common interfacial inhibitor paradigm for ternary trapping of the topoisomerase I-DNA covalent complex. Mol. Cancer Ther. 5, 287-295 (2006).

57 Belmokhtar, C. A., Hillion, J. \& Ségal-Bendirdjian, E. Staurosporine induces apoptosis through both caspase-dependent and caspase-independent mechanisms. Oncogene 20, 3354-3362 (2001).

58 Zhang, X. D., Gillespie, S. K. \& Hersey, P. Staurosporine induces apoptosis of melanoma by both caspase-dependent and -independent apoptotic pathways. Mol. Cancer Ther. 3, 187-197 (2004).

59 Robey, R. W., Steadman, K. \& Polgar, O. Pheophorbide a is a specific probe for ABCG2 function and inhibition. Cancer Res. 64, 1242 (2004).

60 Robey, R. W. et al. Inhibition of ABCG2-mediated transport by protein kinase inhibitors with a bisindolylmaleimide or indolocarbazole structure. Mol. Cancer Ther. 6, 1877-1885 (2007). 